\title{
Multi-object spectroscopy of low redshift EIS clusters. I.`
}

\author{
L. Hansen, L. F. Olsen, and H. E. Jørgensen \\ Copenhagen University Astronomical Observatory, Juliane Maries Vej 30, 2100 Copenhagen, Denmark \\ Received 21 December 2001 / Accepted 5 March 2002

\begin{abstract}
We report the results of the first multi-object spectroscopic observations at the Danish $1.54 \mathrm{~m}$ telescope at La Silla, Chile. Observations of five cluster candidates from the ESO Imaging Survey Cluster Candidate Catalog are described. From these observations we confirm the reality of the five clusters with measured redshifts of $0.11 \leq z \leq 0.35$. We estimate velocity dispersions in the range $294-621 \mathrm{~km} \mathrm{~s}^{-1}$ indicating rather poor clusters. This, and the measured cluster redshifts are consistent with the results of the matched filter procedure applied to produce the Cluster Candidate Catalog.
\end{abstract}

Key words. cosmology: observations - galaxies: distances and redshifts - galaxies: clusters: general

\section{Introduction}

The evolution in the properties of galaxy clusters and their constituent galaxies is an important issue of contemporary cosmology and astrophysics. The demand for large samples of clusters at various redshifts has prompted major systematic efforts to provide catalogues of distant clusters (for references see e.g. Holden et al. 1999). Olsen et al. (1999a, 1999b) and Scodeggio et al. (1999) presented the ESO Imaging Survey (EIS) Cluster Candidate Catalog containing a total of 302 cluster candidates with estimated redshifts of $0.2 \leq z \leq 1.3$. Their work is based on the EIS $I$-band images covering a total area of 17 square degrees. The candidates were selected via a matched filter algorithm akin to the one introduced by Postman et al. (1996).

In order to confirm the reality of the EIS cluster candidates and determine membership of the clusters Ramella et al. (2000) have initiated multi-object spectroscopy (MOS) with the $3.6 \mathrm{~m}$ ESO telescope at La Silla, Chile, for the $0.5 \leq z \leq 0.7$ part of the sample. Furthermore, the spectroscopic investigation of the high redshift candidates $(0.8 \leq z \leq 1.3)$ using the VLT has been initiated by Benoist et al. (2001). They report the detection of bound systems in 3 candidate fields. One of the systems coincides with an X-ray source recently detected by XMM (Neumann et al. 2002).

Here we present the first results for the low redshift part $(0.2 \leq z \leq 0.4)$ based on MOS obtained at the Danish $1.54 \mathrm{~m}$ telescope at La Silla. The data were taken as test observations aiming at implementing MOS at this telescope. The observations were performed during a run in

Send offprint requests to: L. Hansen,

e-mail: leif@astro.ku.dk

* Based on observations made with the Danish 1.5-m telescope at ESO, La Silla, Chile.
February/March 2001 when moon or weather prevented the photometric main program, and exposures through the eight available slit masks covering five cluster candidates (Table 1) were secured. Unfortunately an arrangement suitable for proper flat fielding of the multi-slit images was not yet installed. Flat fields against a screen fixed in the dome were obtained instead, but as described below this procedure did not optimize the quality of the final spectra. Nevertheless, seven masks out of eight had spectra adequate for redshift determination (Table 2).

\section{Observations}

The Danish Faint Object Spectrograph and Camera (DFOSC) mounted at the Danish $1.54 \mathrm{~m}$ telescope is well suited for MOS on galaxy clusters of moderate redshift. Multi-slit masks can be placed in the slit wheel which is easily accessible, and the field is as large as $13.7 \times 13 ! 7$ matching the extent of the moderate redshift clusters. A challenge is the small scale in the telescope focal plane. The slit width used is $2^{\prime \prime}$ or only $128 \mu \mathrm{m}$. The length of the slits varied depending on the extent of the galaxies.

The multi-slits are milled in thin $(0.85 \mathrm{~mm})$ plastic sheets of selected quality in the workshop of the Copenhagen University Astronomical Observatory. The positions and lengths of the slits are determined from preimaging frames of the fields using software that converts the positions on the chip to positions on the slit mask. The transformations are found using a mask with an accurately known grid of pinholes. An exposure through this mask is obtained, and the positions of the images of the pinholes are measured. This procedure has been carried out for two pinhole-masks (P. Kjærgaard, private communication). The accuracies of the two transformations are $2.5 \mu \mathrm{m}$ and $5.3 \mu \mathrm{m}$, corresponding to $0^{\prime \prime} 04$ and $0^{\prime \prime} 08 \mathrm{rms}$, respectively, which gives an estimate of the positional 
accuracy of the slits. The software also allows the observer to avoid overlapping spectra by displaying the computed positions of the spectra on the chip. For aligning the slit mask with the sky, holes corresponding to the position of at least three stars are made.

Alignment of the mask with the sky is carried out using direct exposures of the cluster field and of the slit mask without grism. Offset and rotation are computed based on the positions of the stars and the corresponding holes in the mask using software developed by the local staff (Jones et al. 2001). The field and mask are aligned by offset of the telescope, rotation of DFOSC and possibly fine rotation of the filter wheel. The fine rotation is necessary, because DFOSC cannot be rotated with the required accuracy.

All spectra were obtained with grism \#4 giving a dispersion of $220 \AA / \mathrm{mm}$. The final spectra were extracted in the region $3800-7500 \AA$, but the useful range depended on the spectrum. The resolution determined from $\mathrm{He}-\mathrm{Ne}$ line spectra is found to be $16.6 \AA \mathrm{FWHM}$. Total exposure times of minimum 1 hour were aimed at for each mask, but the images were read out each 20 min to facilitate removal of cosmic ray events in the images.

For the present observations a sample of 5 cluster candidates (Table 1) were selected from Table 1 of Scodeggio et al. (1999). All of these have well developed red sequences (Olsen 2000). The target galaxies were selected along the red sequence, i.e. cluster galaxies of early morphological types are favored. A mask typically contains $\approx 20$ slits, and one or two masks per field covered most of our candidate objects.

\section{Data reduction}

The data reduction was performed using the $\operatorname{IRAF}^{1}$ package. Bias was determined from overscan regions and subtracted. After flat fielding with the dome flats the resulting images were examined for residual fringing, resulting from shifts of the positions of the slit images between science exposures and flat fields. These shifts originate in instrument flexure, that causes the slit images to move slightly with position on the sky.

The flexure induced image shifts are apparent from exposure to exposure even near zenith. As mentioned above flat fields had to be obtained against a screen fixed on the dome, which led to typical shifts of 2-4 pixels between science exposures and exposures on the screen. This is unfortunate because the red part of the flat fields show high frequency variations caused by fringing in the thinned and backside illuminated EEV/MAT chip used. The peak-topeak distance between fringes is only $\approx 15$ pixels. Even small shifts between flat field and science exposures will therefore show residual fringing in the spectra compromising faint spectra. The present data from the test observations are, therefore, not of an optimum quality, and all

\footnotetext{
${ }^{1}$ IRAF is distributed by the National Optical Astronomy Observatories, which is operated by AURA Inc. under contract with NSF.
}
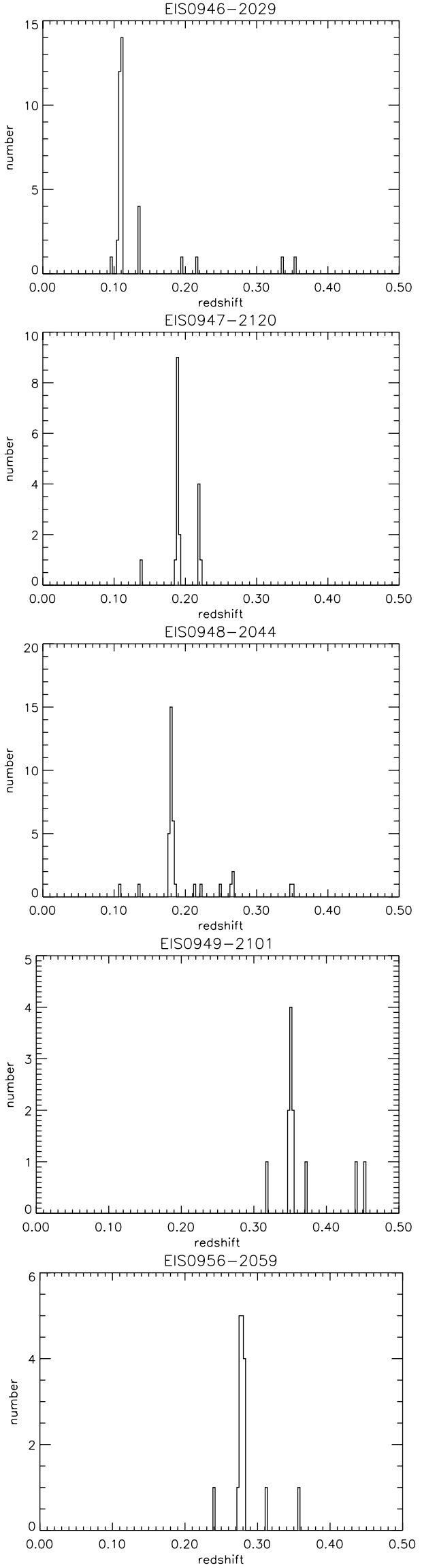

Fig. 1. The distribution of redshifts determined in the 5 cluster fields as indicated in each panel. 
Table 1. Observed cluster candidates. The IDs refer to the EIS Cluster Candidate Catalogue (Scodeggio et al. 1999). $z_{\mathrm{mf}}$ is the matched filter estimated redshift. $\Lambda_{\mathrm{cl}}$ is a parameter of the matched filter method measuring cluster richness. $N_{\mathrm{R}}$ is an Abell-like richness parameter.

\begin{tabular}{lllllll}
\hline \hline Field & $\alpha_{\mathrm{J} 2000}$ & $\delta_{\mathrm{J} 2000}$ & $z_{\mathrm{mf}}$ & $\Lambda_{\mathrm{cl}}$ & $N_{\mathrm{R}}$ & mask \\
\hline EIS0946-2029 & $09: 46: 12.8$ & $-20: 29: 49.6$ & 0.2 & 59.5 & 44 & $11 \& 12$ \\
EIS0947-2120 & $09: 47: 06.9$ & $-21: 20: 55.6$ & 0.2 & 43.4 & 50 & $13 \& 14$ \\
EIS0948-2044 & $09: 48: 07.9$ & $-20: 44: 31.2$ & 0.2 & 42.8 & 32 & $15 \& 16$ \\
EIS0949-2101 & $09: 49: 22.1$ & $-21: 01: 47.1$ & 0.3 & 37.7 & 57 & 18 \\
EIS0956-2059 & $09: 56: 25.2$ & $-20: 59: 49.8$ & 0.3 & 39.7 & 37 & 17 \\
\hline
\end{tabular}

frames through mask \#14 were abandoned because of a too large shift of the flat field. The He-Ne exposures on the other hand were taken before and after the science exposures without changing telescope pointing. Relative flexure shifts were therefore small and could be taken into account.

It is worth mentioning that since the test observations a flat field lamp has been installed in the telescope skybaffle besides the He and Ne lamps, allowing flat fields to be obtained at the same telescope position as the science frames. This procedure is expected to improve the quality of the flat fielding for future MOS observations.

Having discarded the frames with strong residual fringing the remaining frames were stacked using an IRAF script developed for this purpose. After applying small shifts to account for the different positions of the slit images in the science exposures, the script corrects for cosmic ray events and known defects of the chip.

The individual spectra were extracted and wavelength calibrated using the standard IRAF tasks. First the image section containing the two-dimensional spectrum of a slit was copied and wavelength calibrated by means of the corresponding He-Ne spectrum. Then the image section was transformed to wavelength scale along the columns. The background was determined and then subtracted along each individual line, and a one-dimensional spectrum was finally extracted. An approximate flux calibration was performed based on observations of a few spectrophotometric standards observed through a $10^{\prime \prime}$ slit.

Possible residuals from strong sky emission lines were removed by simple interpolation across the lines. A correction for the atmospheric $B$-band absorption around $6870 \AA$ was performed by multiplying with a band correction function. This function was determined from spectrophotometric standards observed with a $2^{\prime \prime}$ slit. The otherwise smooth spectrum was interpolated across the band, and the result divided with the original spectrum.

The redshift determination was performed by Fourier cross-correlation of the spectra with template spectra. The templates were obtained from Kinney et al. (1996) and are UV-optical spectra covering from 1200 to $10000 \AA$. We used template spectra for elliptical, bulge, S0, Sa, Sb, and Sc. The Sc template has very strong emission lines and was suitable for cross correlation with emission line objects. The cross-correlation was performed using the IRAF task $f x c o r$, and results are presented in Tables A.1 through A.5.
The templates were always redshifted close to the redshift under consideration. Whenever a peak in the correlation function was accepted as real or possibly real, the observed spectrum was inspected and compared to the expected positions of the most prominent spectral features. We demanded that some features like the $\mathrm{H}$ and $\mathrm{K}$ lines, the $4000 \AA$ break, or emission lines should be identified before a determination was accepted as certain. This precaution was due to the possibility that residual fringing could introduce spurious correlation peaks. If no convincing features were found, but the correlation peak appeared real, we mark the $z$-value with a colon in Tables A.1A.5. If emission lines are identified the value is marked with an "e". In a number of cases the spectrum suffered from residual fringing or was too weak to yield a redshift determination.

The accuracy of the measured redshifts are influenced by the limited resolution, signal-to-noise, fringing, possible systematic errors in the wavelength transformation, and the consistency with the template. These error sources vary from spectrum to spectrum. A hint of the accuracy is given by the typical FWHM $\Delta z \approx 0.004$ for the Gauss fit to the correlation peak. The position of the peak is likely to be found with an accuracy $\approx 10 \%$ of the FWHM giving an expected standard deviation of $\sigma_{z} \approx 0.0004$. Another way to estimate the error is by comparing the redshift estimates obtained for the same galaxy using different template spectra. From applying all the templates to the same galaxy spectrum we obtain a standard deviation $\sigma_{z}=0.0006$ of the derived redshifts. This is consistent with the error estimated from the width of the correlation peak. Particularly, since some systematic differences between the redshifts derived from the different templates was observed. E.g. the bulge spectrum has a tendency to give slightly higher values than the others. All together we estimate an error for the mean value of $\sigma_{z} \approx 0.0005$.

Only one object has been observed through different masks, namely EIS0948-2044 \#30 for which spectra are available from masks \#15 and \#16. The two results deviate by $\sigma_{z}=0.0006$ consistent with the above estimate.

\section{Results}

Tables A.1 through A.5 give the measured redshifts for all the galaxies. The positions and photometry are from Benoist et al. (1999) and da Costa et al. (2000). 
Table 2. The total integration time (seconds), number of slits, number of successful redshift determinations, and remarks for the 8 MOS masks.

\begin{tabular}{lllrl}
\hline \hline Mask & exp. & $N_{\text {slits }}$ & $N_{\mathrm{z}}$ & remarks \\
\hline 11 & 3900 & 19 & 21 & 3 in one slit \\
12 & 3600 & 18 & 17 & \\
13 & 6000 & 24 & 18 & \\
14 & 3600 & 23 & 0 & no flat field \\
15 & 7200 & 24 & 21 & \\
16 & 7200 & 22 & 17 & \\
17 & 4800 & 20 & 19 & \\
18 & 6000 & 25 & 12 & \\
\hline
\end{tabular}

Magnitudes are total magnitudes determined by SExtractor (MAG_AUTO, Bertin \& Arnouts 1996), and additionally corrected for interstellar extinction as described by Olsen (2000).

We have secured between 12 and 38 redshifts per cluster field. The redshift histograms for each field are shown in Fig. 1. In all fields an obvious peak in the distribution is found indicating the presence of a cluster.

The cluster redshifts are determined as the mean value for all objects in the peak. Firstly, we identify cluster members by accepting all galaxies within $\Delta z \leq 0.01$ from the mean redshift. Then we apply a sigma-clipping at $3.5 \sigma$ to discard outliers, and the redshift of the cluster is determined as the mean of the redshifts of the remaining member galaxies. The cluster members are those galaxies with their redshift printed in boldface in Tables A.1-A.5. We note the high success rate in picking out cluster members which is due to our selection of objects falling on the red sequence.

Table 3 gives the cluster redshifts, $z_{\mathrm{cl}}$, the observed standard deviation in redshift among the cluster members, $\sigma_{\mathrm{cl}}$, the line-of-sight velocity dispersion, $V_{\mathrm{r}}$, and the number of member galaxies found in our samples. The measured cluster redshifts are in good agreement with the matched filter estimated values $\left(z_{\mathrm{mf}}\right.$ in Table 1$) . V_{\mathrm{r}}$ is derived from $\sigma_{\mathrm{cl}}$ after correction for an assumed measuring uncertainty $\sigma_{z}=0.0005$ and has been transferred to the cluster rest frame by division with $1+z$. We find lineof-sight velocity dispersions in the range $294-621 \mathrm{~km} \mathrm{~s}^{-1}$, which is comparable to that of relatively poor clusters of galaxies in the Abell catalog and in agreement with the estimated richness classes of the EIS cluster candidates of $R=0-1$ (Olsen 2000).

In three fields secondary peaks due to possible background concentrations are identified. These are in the fields of EIS0946-2029 ( 4 objects, $z=0.1361 \pm 0.0002$ ), EIS0947-2120 (5 objects, $z=0.2215 \pm 0.0003)$, and EIS0948-2044 (3 objects, $z=0.2671 \pm 0.0004 ; 2$ objects, $z=0.3510 \pm 0.0002)$. Assuming that these concentrations are bound systems the quoted errors can be used to derive an upper limit on the accuracy of the individual measurements, by adopting a negligible velocity dispersion of the systems. From these four systems we estimate the error of an individual redshift measurement to be in the range
Table 3. Observed cluster redshift, observed dispersion in redshift, corrected line-of-sight velocity dispersion $\left(\mathrm{km} \mathrm{s}^{-1}\right)$, and the number of observed members.

\begin{tabular}{lcccc}
\hline \hline Cluster & $z_{\mathrm{cl}}$ & $\sigma_{\mathrm{cl}}$ & $V_{\mathrm{r}}$ & objects \\
\hline EIS0946-2029 & 0.1107 & 0.00172 & 445 & 28 \\
EIS0947-2120 & 0.1910 & 0.00127 & 294 & 12 \\
EIS0948-2044 & 0.1818 & 0.00168 & 407 & 27 \\
EIS0949-2101 & 0.3528 & 0.00247 & 537 & 08 \\
EIS0956-2059 & 0.2797 & 0.00270 & 621 & 15 \\
\hline
\end{tabular}

$\sigma_{z}=0.0003-0.0008$, which supports the previous estimated accuracy of $\sigma_{z} \approx 0.0005$.

\section{Conclusions}

In this work we demonstrate that MOS is possible at the Danish $1.54 \mathrm{~m}$ telescope notwithstanding the high mechanical requirements due to the small image scale. Flat fielding of MOS observations was not optimum during the test observations, but subsequent installation of a flat field lamp in the skybaffle allows future flat fields to be obtained at the same telescope position as the science exposure. The new procedure is expected to yield flat fielding of good quality. In spite of the flat field problems we were able to determine redshifts for 124 galaxies from the test data. We estimate the accuracy of the measured redshifts to be $\sigma_{z} \approx 0.0005$.

The test observations were targeting the fields of 5 EIS cluster candidates selected for their prominent red sequences. Multi-slit masks were constructed to cover members of the red sequences, which leads to a high success rate in picking out cluster members, yielding well-defined cluster redshifts as well as velocity dispersions.

In addition to determining cluster redshifts the data can be used to investigate the quality of the cluster characteristics listed in the EIS Cluster Candidate Catalog (Olsen et al. 1999a, 1999b; Scodeggio et al. 1999). Firstly, we find that the measured redshifts are in good agreement with those originally estimated from the matched filter procedure. Secondly, we note that the derived velocity dispersions are consistent with the clusters being of Abell richness class $R=0-1$ as estimated for the EIS cluster candidates. Both of these results show that the EIS clusters are well-described by their characteristics in the EIS Cluster Candidate Catalog.

The results obtained in this work indicate that MOS on clusters with redshifts up to $z \approx 0.4$ is within reach with the Danish $1.54 \mathrm{~m}$ telescope at La Silla. Furthermore, the confirmation of 5 EIS clusters strongly supports the reality of the EIS cluster candidates. The observations reported here are the first in a systematic program of spectroscopic investigations of the low-redshift sample of the EIS cluster candidates.

Acknowledgements. We thank John Pritchard for making the pre-imaging observations of the fields. We would also like to thank the $2 \mathrm{p} 2$ team, La Silla, for their support, particularly 
Emanuela Pompei for her important help in the development of the alignment software. We are in debt to Michael I. Andersen, Per Kjærgaard, Morten Liborius Jensen, and Anton Norup Sørensen for their great work in developing the MOS mode for DFOSC. We thank the referee Dr. P. Katgert for useful comments. This work has been supported by The Danish Board for Astronomical Research. LFO thanks the Carlsberg Foundation for financial support.

\section{Appendix A: Measured redshifts}

Table A.1. Redshifts obtained in the EIS0946-2029 field. A cluster redshift $z_{\mathrm{cl}}=0.1107$ is determined. Redshifts for accepted members, i.e. deviating less than $3.5 \times \sigma_{\mathrm{cl}}$ (Table 3), are printed in boldface. Whenever no convincing spectral features could be identified despite of a clear correlation peak the value is followed by a colon. An "e" marks objects with identified emission lines.

\begin{tabular}{rccccc}
\hline \hline & $\alpha_{\mathrm{J}} 2000$ & $\delta_{\mathrm{J} 2000}$ & $V$ & $V-I$ & $z$ \\
\hline 1 & $09: 45: 43.9$ & $-20: 31: 51$ & 19.73 & 1.12 & $\mathbf{0 . 1 1 0 5 :}$ \\
2 & $09: 45: 46.2$ & $-20: 31: 43$ & 18.74 & 1.08 & $\mathbf{0 . 1 1 0 6}$ \\
3 & $09: 45: 47.4$ & $-20: 33: 17$ & 20.96 & 1.35 & 0.1975 \\
4 & $09: 45: 49.4$ & $-20: 30: 27$ & 19.42 & 1.14 & 0.2180 \\
5 & $09: 45: 51.1$ & $-20: 28: 03$ & 18.82 & 1.14 & $\mathbf{0 . 1 1 0 5}$ \\
6 & $09: 45: 51.7$ & $-20: 32: 58$ & 17.59 & 1.24 & $\mathbf{0 . 1 1 3 6 e}$ \\
7 & $09: 45: 55.2$ & $-20: 28: 21$ & 18.19 & 1.20 & 0.0000 \\
8 & $09: 46: 00.4$ & $-20: 33: 32$ & 19.58 & 1.12 & $\mathbf{0 . 1 1 1 4}$ \\
9 & $09: 46: 00.7$ & $-20: 32: 56$ & 18.13 & 1.28 & $\mathbf{0 . 1 1 0 3}$ \\
10 & $09: 46: 02.1$ & $-20: 32: 38$ & 19.71 & 1.15 & $\mathbf{0 . 1 1 2 3}$ \\
11 & $09: 46: 03.0$ & $-20: 31: 12$ & 17.09 & 1.26 & $\mathbf{0 . 1 0 7 3}$ \\
12 & $09: 46: 04.3$ & $-20: 33: 22$ & 20.58 & 1.21 & $0.3370:$ \\
13 & $09: 46: 05.3$ & $-20: 29: 14$ & 18.44 & 1.34 & $\mathbf{0 . 1 1 1 4}$ \\
14 & $09: 46: 05.8$ & $-20: 32: 35$ & 19.48 & 1.16 & $\mathbf{0 . 1 1 2 8}$ \\
15 & $09: 46: 07.8$ & $-20: 33: 47$ & 18.27 & 1.21 & $0.1359 \mathrm{e}$ \\
16 & $09: 46: 10.5$ & $-20: 29: 27$ & 18.28 & 1.24 & $\mathbf{0 . 1 1 2 0}$ \\
17 & $09: 46: 11.5$ & $-20: 29: 26$ & 17.14 & 1.25 & $\mathbf{0 . 1 1 1 2}$ \\
18 & $09: 46: 11.9$ & $-20: 31: 40$ & 19.49 & 1.27 & $\mathbf{0 . 1 1 1 2}$ \\
19 & $09: 46: 12.4$ & $-20: 29: 28$ & 17.55 & 1.27 & $\mathbf{0 . 1 1 1 9}$ \\
20 & $09: 46: 13.8$ & $-20: 29: 53$ & 19.23 & 1.32 & 0.3550 \\
21 & $09: 46: 14.3$ & $-20: 28: 35$ & 17.90 & 1.26 & $\mathbf{0 . 1 1 2 4}$ \\
22 & $09: 46: 16.2$ & $-20: 33: 55$ & 18.53 & 1.24 & $\mathbf{0 . 1 0 8 2}$ \\
23 & $09: 46: 18.0$ & $-20: 26: 59$ & 19.57 & 1.34 & $\mathbf{0 . 1 0 8 5}$ \\
24 & $09: 46: 18.8$ & $-20: 30: 35$ & 17.99 & 1.12 & $\mathbf{0 . 1 0 8 4}$ \\
25 & $09: 46: 21.5$ & $-20: 33: 51$ & 18.57 & 1.19 & $\mathbf{0 . 1 1 0 9}$ \\
26 & $09: 46: 22.1$ & $-20: 27: 45$ & 19.56 & 1.17 & $\mathbf{0 . 1 1 0 0}$ \\
27 & $09: 46: 22.5$ & $-20: 28: 11$ & 17.88 & 1.21 & $\mathbf{0 . 1 0 9 8}$ \\
28 & $09: 46: 24.3$ & $-20: 31: 06$ & 17.47 & 1.23 & $\mathbf{0 . 1 0 8 1 e}$ \\
29 & $09: 46: 24.5$ & $-20: 31: 19$ & 19.08 & 1.17 & $\mathbf{0 . 1 1 1 2}$ \\
30 & $09: 46: 25.4$ & $-20: 28: 40$ & 19.64 & 1.14 & $\mathbf{0 . 1 1 3 9}$ \\
31 & $09: 46: 26.2$ & $-20: 26: 43$ & 18.47 & 1.24 & $\mathbf{0 . 1 0 7 8}$ \\
32 & $09: 46: 28.4$ & $-20: 33: 56$ & 20.27 & 1.18 & $0.1357:$ \\
33 & $09: 46: 29.3$ & $-20: 33: 32$ & 18.68 & 1.12 & $\mathbf{0 . 1 1 1 1}$ \\
34 & $09: 46: 29.9$ & $-20: 33: 52$ & 18.60 & 1.13 & $0.1362 \mathrm{e}$ \\
35 & $09: 46: 30.7$ & $-20: 26: 17$ & 18.88 & 1.33 & $0.0980 \mathrm{e}$ \\
36 & $09: 46: 32.6$ & $-20: 33: 29$ & 17.40 & 1.07 & 0.1365 \\
37 & $09: 46: 33.1$ & $-20: 29: 24$ & 19.36 & 1.09 & $\mathbf{0 . 1 1 0 5}$ \\
38 & $09: 46: 36.7$ & $-20: 28: 18$ & 16.55 & 1.10 & $\mathbf{0 . 1 1 2 1}$ \\
\hline & & & & &
\end{tabular}

Table A.2. Redshifts obtained in the EIS0947-2120 field. A cluster redshift of $z_{\mathrm{cl}}=0.1910$ is determined.

\begin{tabular}{rccccc}
\hline \hline & $\alpha_{\mathrm{J} 2000}$ & $\delta_{\mathrm{J} 2000}$ & $V$ & $V-I$ & $z$ \\
\hline 1 & $09: 46: 43.2$ & $-21: 20: 34$ & 19.61 & 1.33 & $\mathbf{0 . 1 9 1 9 :}$ \\
2 & $09: 46: 46.6$ & $-21: 21: 26$ & 19.25 & 1.39 & $\mathbf{0 . 1 9 1 2}$ \\
3 & $09: 46: 51.8$ & $-21: 20: 18$ & 19.00 & 1.47 & $\mathbf{0 . 1 9 1 1}$ \\
4 & $09: 46: 53.0$ & $-21: 24: 54$ & 18.33 & 1.44 & 0.1402 \\
5 & $09: 46: 53.4$ & $-21: 18: 27$ & 20.31 & 1.46 & $\mathbf{0 . 1 8 9 5}$ \\
6 & $09: 46: 57.1$ & $-21: 21: 04$ & 19.01 & 1.46 & $\mathbf{0 . 1 9 2 5}$ \\
7 & $09: 46: 58.1$ & $-21: 21: 39$ & 19.40 & 1.43 & $\mathbf{0 . 1 9 1 2}$ \\
8 & $09: 47: 00.3$ & $-21: 23: 06$ & 19.04 & 1.52 & $\mathbf{0 . 1 8 8 9}$ \\
9 & $09: 47: 01.7$ & $-21: 22: 26$ & 19.45 & 1.60 & 0.2205 \\
10 & $09: 47: 03.2$ & $-21: 20: 54$ & 18.65 & 1.43 & $\mathbf{0 . 1 9 0 6}$ \\
11 & $09: 47: 04.6$ & $-21: 19: 20$ & 19.41 & 1.43 & $\mathbf{0 . 1 8 9 6}$ \\
12 & $09: 47: 05.9$ & $-21: 20: 08$ & 17.65 & 1.42 & $\mathbf{0 . 1 9 1 1}$ \\
13 & $09: 47: 07.2$ & $-21: 21: 39$ & 18.90 & 1.56 & $\mathbf{0 . 1 9 3 4}$ \\
14 & $09: 47: 10.6$ & $-21: 23: 53$ & 19.56 & 1.51 & $\mathbf{0 . 1 9 0 7}$ \\
15 & $09: 47: 25.8$ & $-21: 20: 11$ & 19.92 & 1.54 & $0.2214:$ \\
16 & $09: 47: 28.4$ & $-21: 22: 54$ & 18.98 & 1.52 & 0.2226 \\
17 & $09: 47: 29.9$ & $-21: 22: 29$ & 19.94 & 1.59 & 0.2215 \\
18 & $09: 47: 35.4$ & $-21: 23: 38$ & 18.67 & 1.47 & 0.2215 \\
\hline
\end{tabular}

Table A.3. Redshifts obtained in the EIS0948-2044 field. A cluster redshift of $z_{\mathrm{cl}}=0.1818$ is determined.

\begin{tabular}{rccccc}
\hline \hline & $\alpha_{\mathrm{J} 2000}$ & $\delta_{\mathrm{J} 2000}$ & $V$ & $V-I$ & $z$ \\
\hline 1 & $09: 47: 45.3$ & $-20: 41: 41$ & 18.31 & 1.50 & $\mathbf{0 . 1 8 2 6}$ \\
2 & $09: 47: 45.3$ & $-20: 42: 24$ & 20.20 & 1.33 & $0.2497:$ \\
3 & $09: 47: 52.8$ & $-20: 46: 36$ & 20.37 & 1.38 & $\mathbf{0 . 1 8 1 6}$ \\
4 & $09: 47: 54.2$ & $-20: 41: 23$ & 18.59 & 1.43 & $\mathbf{0 . 1 8 0 5}$ \\
5 & $09: 47: 55.6$ & $-20: 43: 49$ & 19.48 & 1.44 & 0.2225 \\
6 & $09: 47: 56.4$ & $-20: 44: 21$ & 19.21 & 1.40 & $\mathbf{0 . 1 8 3 8}$ \\
7 & $09: 47: 56.5$ & $-20: 40: 47$ & 18.09 & 1.49 & $\mathbf{0 . 1 8 1 8}$ \\
8 & $09: 47: 56.8$ & $-20: 41: 53$ & 20.84 & 1.42 & $\mathbf{0 . 1 8 0 0}$ \\
9 & $09: 47: 57.8$ & $-20: 42: 39$ & 19.34 & 1.47 & $\mathbf{0 . 1 8 4 2}$ \\
10 & $09: 47: 58.9$ & $-20: 44: 18$ & 21.20 & 1.27 & $\mathbf{0 . 1 7 9 2}$ \\
11 & $09: 47: 59.6$ & $-20: 42: 13$ & 19.89 & 1.39 & $\mathbf{0 . 1 8 1 3}$ \\
12 & $09: 48: 00.5$ & $-20: 43: 14$ & 19.82 & 1.36 & $\mathbf{0 . 1 7 9 0}$ \\
13 & $09: 48: 03.6$ & $-20: 45: 51$ & 19.11 & 1.35 & $\mathbf{0 . 1 8 4 7}$ \\
14 & $09: 48: 05.2$ & $-20: 43: 46$ & 18.04 & 1.50 & $\mathbf{0 . 1 8 3 0}$ \\
15 & $09: 48: 05.2$ & $-20: 44: 32$ & 19.44 & 1.48 & $\mathbf{0 . 1 8 2 4}$ \\
16 & $09: 48: 05.8$ & $-20: 43: 57$ & 19.69 & 1.39 & $\mathbf{0 . 1 8 1 2}$ \\
17 & $09: 48: 06.5$ & $-20: 42: 53$ & 19.63 & 1.39 & $\mathbf{0 . 1 8 7 1}$ \\
18 & $09: 48: 08.6$ & $-20: 44: 03$ & 19.14 & 1.39 & $\mathbf{0 . 1 8 3 3}$ \\
19 & $09: 48: 08.7$ & $-20: 45: 00$ & 19.68 & 1.40 & $\mathbf{0 . 1 8 1 7}$ \\
20 & $09: 48: 09.9$ & $-20: 43: 15$ & 20.63 & 1.49 & 0.2158 \\
21 & $09: 48: 10.4$ & $-20: 45: 22$ & 19.04 & 1.51 & $\mathbf{0 . 1 8 2 1}$ \\
22 & $09: 48: 11.1$ & $-20: 45: 30$ & 19.49 & 1.54 & $0.3512 \mathrm{e}$ \\
23 & $09: 48: 11.4$ & $-20: 45: 33$ & 20.29 & 1.41 & $0.3508 \mathrm{e}$ \\
24 & $09: 48: 12.4$ & $-20: 48: 49$ & 19.31 & 1.33 & $\mathbf{0 . 1 8 3 0 :}$ \\
25 & $09: 48: 12.4$ & $-20: 43: 14$ & 21.02 & 1.33 & $0.1372:$ \\
26 & $09: 48: 14.4$ & $-20: 46: 48$ & 19.60 & 1.34 & $\mathbf{0 . 1 8 4 3}$ \\
27 & $09: 48: 14.8$ & $-20: 45: 23$ & 18.90 & 1.44 & $\mathbf{0 . 1 8 3 5}$ \\
28 & $09: 48: 16.5$ & $-20: 44: 32$ & 19.73 & 1.42 & $\mathbf{0 . 1 8 1 4}$ \\
29 & $09: 48: 17.1$ & $-20: 42: 28$ & 18.51 & 1.26 & 0.1107 \\
30 & $09: 48: 20.7$ & $-20: 45: 16$ & 20.29 & 1.53 & 0.2662 \\
31 & $09: 48: 22.1$ & $-20: 48: 13$ & 18.29 & 1.26 & $\mathbf{0 . 1 7 9 8}$ \\
32 & $09: 48: 23.7$ & $-20: 44: 32$ & 20.87 & 1.39 & $\mathbf{0 . 1 8 1 6}$ \\
33 & $09: 48: 24.5$ & $-20: 44: 38$ & 20.02 & 1.55 & 0.2676 \\
34 & $09: 48: 24.8$ & $-20: 45: 24$ & 20.63 & 1.26 & $\mathbf{0 . 1 7 9 8}$ \\
35 & $09: 48: 26.2$ & $-20: 48: 03$ & 19.88 & 1.41 & $\mathbf{0 . 1 7 9 1 e}$ \\
36 & $09: 48: 30.9$ & $-20: 43: 17$ & 20.42 & 1.28 & $\mathbf{0 . 1 8 3 0 :}$ \\
37 & $09: 48: 35.6$ & $-20: 45: 39$ & 20.34 & 1.27 & $0.2674:$ \\
\hline & & & & &
\end{tabular}


Table A.4. Redshifts obtained in the EIS0949-2101 field. A cluster redshift of $z_{\mathrm{cl}}=0.3528$ is determined.

\begin{tabular}{rccccc}
\hline \hline & $\alpha_{\mathrm{J} 2000}$ & $\delta_{\mathrm{J} 2000}$ & $V$ & $V-I$ & $z$ \\
\hline 1 & $09: 49: 02.2$ & $-21: 00: 33$ & 21.36 & 2.14 & $0.4418:$ \\
2 & $09: 49: 05.0$ & $-21: 02: 59$ & 20.38 & 2.17 & $0.4536 \mathrm{e}$ \\
3 & $09: 49: 12.2$ & $-20: 59: 05$ & 21.84 & 2.05 & $0.3730:$ \\
4 & $09: 49: 13.4$ & $-20: 59: 25$ & 21.69 & 2.13 & $\mathbf{0 . 3 5 0 0}$ \\
5 & $09: 49: 15.8$ & $-20: 59: 52$ & 20.92 & 2.03 & $\mathbf{0 . 3 4 9 9}$ \\
6 & $09: 49: 19.0$ & $-21: 02: 08$ & 21.30 & 2.05 & $\mathbf{0 . 3 5 3 9}$ \\
7 & $09: 49: 20.0$ & $-21: 01: 07$ & 20.66 & 2.02 & $\mathbf{0 . 3 5 1 0}$ \\
8 & $09: 49: 20.6$ & $-21: 01: 16$ & 19.78 & 1.98 & $\mathbf{0 . 3 5 3 5}$ \\
9 & $09: 49: 23.1$ & $-21: 02: 05$ & 20.32 & 2.10 & $\mathbf{0 . 3 5 2 0}$ \\
10 & $09: 49: 25.1$ & $-21: 02: 19$ & 20.69 & 2.01 & $\mathbf{0 . 3 5 5 5}$ \\
11 & $09: 49: 32.0$ & $-21: 01: 18$ & 21.06 & 2.08 & $\mathbf{0 . 3 5 6 5}$ \\
12 & $09: 49: 42.0$ & $-21: 04: 13$ & 21.52 & 1.92 & 0.3181 \\
\hline
\end{tabular}

Table A.5. Redshifts obtained in the EIS0956-2059 field. A cluster redshift of $z_{\mathrm{cl}}=0.2797$ is determined.

\begin{tabular}{rccccc}
\hline \hline & $\alpha_{\mathrm{J} 2000}$ & $\delta_{\mathrm{J} 2000}$ & $V$ & $V-I$ & $z$ \\
\hline 1 & $09: 56: 12.6$ & $-20: 59: 21$ & 17.23 & 1.62 & 0.0000 \\
2 & $09: 56: 12.8$ & $-20: 57: 10$ & 20.54 & 1.77 & $\mathbf{0 . 2 8 3 1}$ \\
3 & $09: 56: 15.8$ & $-21: 02: 14$ & 20.53 & 1.68 & $\mathbf{0 . 2 8 0 4}$ \\
4 & $09: 56: 17.8$ & $-20: 56: 44$ & 20.07 & 1.84 & 0.3589 \\
5 & $09: 56: 18.9$ & $-20: 58: 55$ & 19.93 & 1.62 & $0.3136:$ \\
6 & $09: 56: 20.6$ & $-20: 57: 36$ & 20.37 & 1.76 & $\mathbf{0 . 2 7 8 7}$ \\
7 & $09: 56: 24.7$ & $-20: 59: 22$ & 19.25 & 1.65 & $\mathbf{0 . 2 8 0 6}$ \\
8 & $09: 56: 25.8$ & $-21: 00: 40$ & 20.08 & 1.79 & $\mathbf{0 . 2 8 0 4}$ \\
9 & $09: 56: 26.6$ & $-21: 00: 16$ & 19.90 & 1.75 & $\mathbf{0 . 2 7 8 4}$ \\
10 & $09: 56: 27.5$ & $-21: 00: 36$ & 20.58 & 1.70 & $\mathbf{0 . 2 7 7 1}$ \\
11 & $09: 56: 28.2$ & $-20: 58: 58$ & 20.55 & 1.71 & $\mathbf{0 . 2 7 3 1}$ \\
12 & $09: 56: 30.1$ & $-21: 01: 39$ & 20.42 & 1.65 & $\mathbf{0 . 2 8 3 7}$ \\
13 & $09: 56: 30.9$ & $-20: 57: 48$ & 19.58 & 1.65 & 0.2423 \\
14 & $09: 56: 32.5$ & $-21: 03: 16$ & 19.90 & 1.75 & $\mathbf{0 . 2 8 2 2}$ \\
15 & $09: 56: 34.3$ & $-20: 58: 44$ & 20.36 & 1.68 & $\mathbf{0 . 2 8 2 1}$ \\
16 & $09: 56: 37.0$ & $-20: 57: 57$ & 19.89 & 1.56 & $\mathbf{0 . 2 7 7 5}$ \\
17 & $09: 56: 37.9$ & $-21: 02: 13$ & 19.19 & 1.71 & $\mathbf{0 . 2 8 0 5}$ \\
18 & $09: 56: 41.8$ & $-21: 03: 18$ & 19.59 & 1.69 & $\mathbf{0 . 2 7 8 3}$ \\
19 & $09: 56: 47.3$ & $-20: 57: 38$ & 20.48 & 1.81 & $\mathbf{0 . 2 7 9 6}$ \\
\hline
\end{tabular}

\section{References}

Benoist, C., da Costa, L., Olsen, L. F., et al. 1999, A\&A, 346, 58

Benoist, C., da Costa, L., Jørgensen, H. E., et al. 2001, preprint to be published in A\&A

Bertin, E., \& Arnouts S. 1996, A\&AS, 117, 393

da Costa, L., Arnouts, S., Benoist, C., et al. 2000, The Messenger, 98, 36

Holden, B. P., Nichol, R. C., Romer, A. K., et al. 1999, AJ, 118, 2002

Jones, H., Pompei, E., and the 2p2 Team 2001, The Messenger, 104,7

Kinney, A. L., Calzetti, D., Bohlin, R. C., et al. 1996, ApJ, 467,38
Neumann, D. M., Benoist, C., Arnaud, M., et al. 2002, to appear in Proc. Symp., New Visions of the X-ray Universe in the XMM-Newton and Chandra Era

Olsen, L. F., Scodeggio, M., da Costa, L., et al. 1999, A\&A, 345,363

Olsen, L. F., Scodeggio, M., da Costa, L., et al. 1999, A\&A, 345,681

Olsen, L. F. 2000, Ph.D.-dissertation

Postman, M., Lubin, L. M., Gunn J. E., et al. 1996, AJ, 111, 615

Ramella, M., Biviano, A., Boschin, W., et al. 2000, A\&A, 360, 861

Scodeggio, M., Olsen, L. F., da Costa, L., et al. 1999, A\&AS, 137,83 